\title{
Simultaneous second-harmonic generation, third-harmonic generation, and four-wave mixing microscopy with single sub-8 fs laser pulses
}

\author{
R. Selm, ${ }^{1}$ G. Krauss, ${ }^{2}$ A. Leitenstorfer, ${ }^{2}$ and A. Zumbusch ${ }^{1, a)}$ \\ ${ }^{1}$ Department of Chemistry, University of Konstanz, D-78457 Konstanz, Germany \\ ${ }^{2}$ Department of Physics, University of Konstanz, D-78457 Konstanz, Germany
}

(Received 8 August 2011; accepted 16 October 2011; published online 4 November 2011)

\begin{abstract}
We demonstrate a compact pulse compression scheme that offers flexible chirp control for improved conversion efficiencies in high resolution nonlinear optical microscopy. An Er:fiber laser combined with a highly nonlinear optical fiber yields pulses centered at $1100 \mathrm{~nm}$ with a bandwidth of $500 \mathrm{~nm}$. The compressed pulses with a duration of 7.6 fs enable simultaneous second-harmonic generation, third-harmonic generation, and four-wave mixing microscopy. The spectrum is tailored for an ideal compromise between conversion efficiency and spectral discrimination between the three types of broadband nonlinear signals. Distinct differences in structural contrast obtained from the simultaneous read-out of the three nonlinear signals are demonstrated in a biological sample. (C) 2011 American Institute of Physics. [doi:10.1063/1.3658456]
\end{abstract}

The ready availability of ultrafast laser sources has recently led to a broad use of nonlinear optical microscopy techniques. They feature inherent 3D sectioning capabilities, and many of them are suited for label-free imaging. Long excitation wavelengths in the near infrared spectral range allow for deep tissue penetration depths. ${ }^{1}$ Second-harmonic generation $(\mathrm{SHG}),{ }^{2-4}$ third-harmonic generation $(\mathrm{THG}),{ }^{4-6}$ and four-wave mixing $(\mathrm{FWM})^{7,8}$ microscopy provide different contrast mechanisms for the visualization of structural or chemical information of unstained biological samples. SHG is observable in non-centrosymmetric media (e.g., collagene) and maps the $\chi^{(2)}$ susceptibility of the sample. THG and FWM, by contrast, are not restricted to asymmetric media and show high contrast for structures with large $\chi^{(3)}$ values such as lipids. ${ }^{6}$ Under tight focusing conditions, the Gouy phase shift must be taken into account. Strong THG signals are therefore generated at interfaces and for small sample features. ${ }^{5,9-13}$ FWM, on the other hand, shows superior phase-matching behavior in bulk media and therefore delivers complementary information. ${ }^{8}$ A prominent special case of FWM is coherent anti-Stokes Raman scattering (CARS) microscopy, which allows for fast chemically selective microscopy. In this case, vibrational resonances are exploited for signal generation. ${ }^{14}$

Commonly, titanium-sapphire oscillators or synchronously pumped optical parametric oscillators with typical pulse durations of $100 \mathrm{fs}$ or longer are used for SHG and THG microscopy. ${ }^{15}$ The efficiencies for the generation of nonlinear signals can be increased by using shorter laser pulses, which have to be achieved at the focal position. ${ }^{16}$

Recently, generation of ultrashort laser pulses with a central wavelength of $1200 \mathrm{~nm}$ was demonstrated by using a mode-locked Er:fiber laser in combination with a highly nonlinear optical fiber. ${ }^{17}$ In this spectral domain, low photodamage to biological samples is expected. ${ }^{18}$ Using all-reflective focusing optics a pulse duration of $7.8 \mathrm{fs}$ was achieved. ${ }^{17}$ For high resolution microscopy in biological samples, however,

\footnotetext{
${ }^{\text {a) }}$ Author to whom correspondence should be addressed. Electronic mail: andreas.zumbusch@uni-konstanz.de.
}

highly corrected microscope objectives with large numerical apertures are required. This necessitates the use of refractive optics, which complicates dispersion compensation. A pulse duration of $11.4 \mathrm{fs}$ in the focus of a $0.85 \mathrm{NA}$ objective has been realized with a simple prism compressor. ${ }^{19}$ Reaching results close to the bandwidth limit, which is important for obtaining high nonlinear conversion efficiencies, is complicated by residual chirp induced by the passage through the glass of the objective. Superior dispersion control may be provided by spatial light modulators such as deformable mirrors, liquid crystal arrays, or acousto-optic modulators. ${ }^{20-22}$ While these are flexible instruments well suited for dispersion compensation, they add significant cost and complexity to the microscope. Here we show how pulse durations of 7.6 fs in the microscope focus can be achieved by a simple split prism compressor scheme. Split prism compressors have been used previously for precompressing the combination of two laser pulses with separated central wavelengths and narrow bandwidth. ${ }^{23}$ With the single ultrashort laser pulses produced in this way, SHG, THG, and FWM images may be recorded simultaneously.

The light source is based on a mode-locked Er:fiber laser at $1550 \mathrm{~nm}$ with a repetition rate of $40 \mathrm{MHz} .{ }^{17}$ The amplified output reaches a pulse energy of $9 \mathrm{~nJ}$ and is compressed to $130 \mathrm{fs}$. In a highly nonlinear fiber of $3 \mathrm{~mm}$ length and zero dispersion wavelength $\lambda_{\mathrm{ZD}}=1.36 \mu \mathrm{m}$ (see Ref. 17 for more details) the fundamental splits into two parts. A solitonic wave shifts towards longer wavelengths and a dispersive wave shifts towards shorter wavelengths. The supercontinuum is tailored such that the SHG, THG, and FWM signals can be detected simultaneously and free of spectral overlap (Fig. 1). The fundamental and solitonic components are blocked by a razor blade in the Fourier plane of the prism compressor [Fig. 2(a)]. The dispersive wave of the fiber output is used for excitation. It has a bandwidth of $500 \mathrm{~nm}$ centered at $1100 \mathrm{~nm}$ [Fig. 2(b)] and an average power of $10 \mathrm{~mW}$ in the microscope focus.

In order to compress the fiber output, we use a prism compressor which is different from conventional schemes [see Fig. 2(a)]. It consists of three NSF10 equilateral prisms 


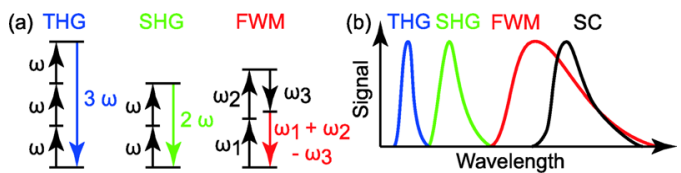

FIG. 1. (Color online) (a) Energy level diagrams, frequency components of supercontinuum pulse $\left(\omega, \omega_{1}, \omega_{2}\right.$, and $\left.\omega_{3}\right)$, nonlinear signals at $3 \omega$ (THG), $2 \omega$ (SHG) and $\omega_{1}+\omega_{2}-\omega_{3}$ (FWM). (b) THG, SHG, and FWM signals free of spectral overlap and supercontinuum pulse (SC).

(P1, P2, and P3), which form two prism pairs with the first prism P1 used jointly. The broad spectrum is refracted by the first prism P1 and dispersed over two separate second prisms P2 and P3. The adjustment of the material passage through the prisms P2 and P3 allows for independent second-order dispersion control of the short and long wavelength parts of the supercontinuum, which are split at the tip of prism P2. In this way, a flat spectral phase can be extended to the wings of the spectrum allowing for a shorter pulse duration. This scheme offers a great advantage over a typical prism compressor where the second-order dispersion is compensated only around one wavelength component. The compressor described has a zero-crossing of the third-order dispersion within the laser spectrum. Therefore, little third-order dispersion is added. One of the end mirrors in the Fourier plane of the compressor is translated to adjust temporal overlap between the two separate spectral parts. Note that small fractions of the supercontinuum are sacrificed at the tip of prism $\mathrm{P} 2$, which results in a dip at $970 \mathrm{~nm}$ in the spectrum [Figs. 2(a) and 2(b)]. This loss is small because the second and third prism P2 and P3 are placed close to the Fourier plane of the compressor with a highly spectrally dispersed and tightly focused beam at the prism tip. Careful fabrication of the prism tip could minimize the loss further. The laser pulse in the focus of a refractive water immersion objective (NA $1.2,63 \times$, Leica, Wetzlar, Germany) is analyzed by a SHG fringe-resolved autocorrelation (FRAC) measurement [Fig. 2(c)]. A simulated FRAC trace based on the spectrum and a polynomial phase fit is shown for comparison. A temporal width of $t_{\mathrm{FWHM}}=(7.6 \pm 1.0) \mathrm{fs}$ is extracted, which is close to the bandwidth limit of $7.2 \mathrm{fs}$ supported by the spectrum.


FIG. 2. (Color online) (a) Pulse compression and microscope setup. EDFA: erbium doped fiber amplifier, HNF: highly nonlinear fiber, P: off-axis paraboloidal mirror, FM: focusing mirror, P1/P2/P3: equilateral NSF10 prism, M1/M2: end mirrors, RB: razor blade, PM: pick-off mirror, T: reflective telescope, O1/O2: focusing/collection objective, PS: xyz-piezo stage, F: emission filter, S: spectrometer. (b) Measured spectrum in the microscope focus and (c) measured FRAC trace (dots) and simulated trace (line) derived from the spectrum with a polynomial phase fit.
Applications of the light source described above are demonstrated by using a home-built sample scanning microscope [Fig. 2(a)] based on a xyz-piezo stage (P-563.3CD, Physik Instrumente, Karlsruhe, Germany). The laser beam is expanded by a reflective telescope before being focused by the water immersion objective. 3D-microscopy is possible by raster scanning the sample through a fixed focus using the piezo stage. The nonlinear coherent signals are detected in the forward direction by a long working distance objective (NA 0.6, 20×, Edmund Optics, Karlsruhe, Germany). The excitation light is filtered by a series of dielectric filters. A prism spectrometer with a CCD camera (iXon ${ }^{\mathrm{EM}}+897$ back illuminated, Andor Technology plc., Belfast, Northern Ireland) is used for spectral image acquisition.

As a first example, simultaneous SHG, THG, and FWM imaging microscopy is demonstrated on zinc oxide particles. Fig. 3(a) shows clusters of $\mathrm{ZnO}$ using the three different imaging modalities. The appearance of the structures is affected by the different phase-matching mechanisms. The $\mathrm{ZnO}$ clusters serve as an ideal sample for the spectral characterization of the microscope setup. In Fig. 3(b), the broadband nonlinear signals are shown on a cluster of $\mathrm{ZnO}$ at the position of the cross in the image. The nonlinear imaging modalities are nicely discernible because the signals are free of spectral overlap. We have verified the different origin of the detected coherent nonlinear signals by measuring their excitation power dependence (data not shown). Due to limitations of the transmission bands of the dielectric emission filters, the THG signal is cut below $390 \mathrm{~nm}$ and the FWM signal is cut above $750 \mathrm{~nm}$. Dedicated filters and single point detectors for the three different channels as well as a beam scanning geometry based on galvanometer driven mirrors for image acquisition would allow for faster imaging.

Our light source is readily applicable to biological investigations. Structural information of Caenorhabditis elegans samples can be extracted by the three different nonlinear optical imaging modes (Fig. 4). The image shows a single section through a $C$. elegans worm in buffer (anesthetized by sodium azide). Although THG and FWM both rely mainly on nonresonant $\chi^{(3)}$ susceptibilities, complementary structural information is obtained using both imaging techniques. THG signals appear brighter for lipid vesicles, whereas FWM microscopy is better suited for imaging diffuse structures. The SHG channel, by contrast, clearly shows the muscular structure of the worm. Here, long and thin collagen fibers can be detected. The last image shows the overlay of all three detection channels. In conclusion, we have presented an ultrashort light source based on a reliable Er:fiber laser. A simple prism compressor scheme for efficient laser
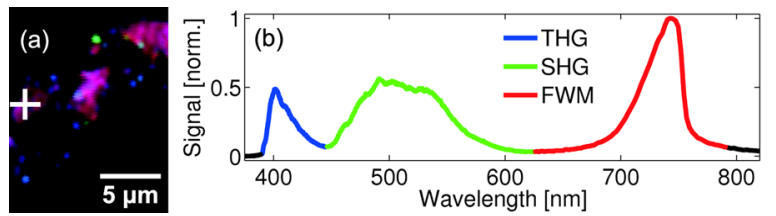

FIG. 3. (Color online) Multimodal microscopy of $\mathrm{ZnO}$ clusters. (a) Composed image: THG, SHG, and FWM, contrast mechanisms depend on the sample's microscopic structure. (b) Peaks in the spectra taken at the position of the cross: left (THG), middle (SHG), right (FWM). Pixel dwell time is $0.4 \mathrm{~ms}, 105$ pixel $\times 135$ pixel. 

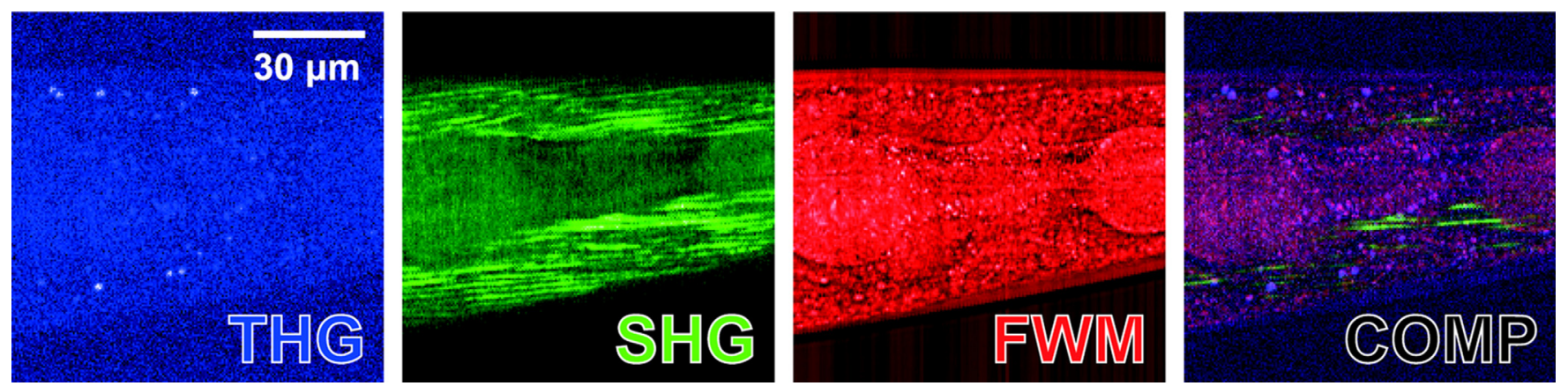

FIG. 4. (Color online) Label-free multimodal microscopy of the pharynx of a C. elegans worm. The THG image distinctly shows the lipid vesicles. The SHG image indicates collagene in the muscle fibers. The FWM reflects the shape around the pharynx of the worm. The image on the right shows the composition (COMP) of all three types of signals. Pixel dwell time is $1 \mathrm{~ms}, 200$ pixel $\times 199$ pixel.

pulse compression is described. This compression scheme is flexible and offers versatile chirp control, e.g., in nonlinear optical microscopes. It can be used to generate sub- 8 fs pulses in the focus of a high numerical aperture water immersion objective. We apply this system to perform labelfree nonlinear imaging with simultaneous detection of three different non-linear optical signals using single laser pulses. As an application we demonstrate simultaneous SHG, THG, and FWM investigations of $C$. elegans.

We thank the group of E. Deuerling at the University of Konstanz for providing the C. elegans samples. Financial support from the Baden-Württemberg Stiftung is gratefully acknowledged.

${ }^{1}$ M. Balu, T. Baldacchini, J. Carter, T. B. Krasieva, R. Zadoyan, and B. J. Tromberg, J. Biomed. Opt. 14, 010508 (2009).

${ }^{2}$ I. Freund, M. Deutsch, and A. Sprecher, Biophys. J. 50, 693 (1986).

${ }^{3}$ P. J. Campagnola, H. A. Clark, W. A. Mohler, A. Lewis, and L. M. Loew, J. Biomed. Opt. 6, 277 (2001).

${ }^{4}$ N. Olivier, M. A. Luengo-Oroz, L. Duloquin, E. Faure, T. Savy, I. Veilleux, X. Solinas, D. Débarre, P. Bourgine, A. Santos, N. Peyriéras, and E. Beaurepaire, Science 329, 967 (2010).

${ }^{5}$ Y. Barad, H. Eisenberg, M. Horowitz, and Y. Silberberg, Appl. Phys. Lett. 70, 922 (1997).

${ }^{6}$ D. Débarre, W. Supatto, A.-M. Pena, A. Fabre, T. Tordjmann, L. Combettes, M.-C. Schanne-Klein, and E. Beaurepaire, Nat. Methods 3, 47 (2006).
${ }^{7}$ A. Zumbusch, G. R. Holtom, and X. S. Xie, Phys. Rev. Lett. 82, 4142 (1999).

${ }^{8}$ W. Min, S. Lu, M. Rueckel, G. R. Holtom, and X. S. Xie, Nano Lett. 9, 2423 (2009).

${ }^{9}$ J. F. Ward and G. H. C. New, Phys. Rev. 185, 57 (1969).

${ }^{10}$ T. Y. F. Tsang, Phys. Rev. A 52, 52 (1995).

${ }^{11}$ J. A. Squier, M. Müller, G. J. Brakenhoff, and K. R. Wilson, Opt. Exp. 3, 315 (1998).

${ }^{12}$ J.-X. Cheng and X. S. Xie, J. Opt. Soc. Am. B 19, 1604 (2002).

${ }^{13}$ D. Débarre, W. Supatto, and E. Beaurepaire, Opt. Lett. 30, 2134 (2005).

${ }^{14}$ M. Müller and A. Zumbusch, ChemPhysChem 8, 2156 (2007).

${ }^{15}$ F. Aptel, N. Olivier, A. Deniset-Besseau, J.-M. Legeais, K. Plamann, M.C. Schanne-Klein, and E. Beaurepaire, Invest. Ophthalmol. Vis. Sci. 51, 2459 (2010).

${ }^{16}$ C. J. Bardeen, V. V. Yakovlev, J. A. Squier, K. R. Wilson, S. D. Carpenter, P. M. Weber, J. Biomed. Opt. 4, 362 (1999).

${ }^{17}$ A. Sell, G. Krauss, R. Scheu, R. Huber, and A. Leitenstorfer, Opt. Exp. 17, 1070 (2009).

${ }^{18}$ I. H. Chen, S. W. Chu, C. K. Sun, P. C. Cheng, and B. L. Lin, Opt. Quantum Electron. 34, 1251 (2002).

${ }^{19}$ R. Selm, M. Winterhalder, A. Zumbusch, G. Krauss, T. Hanke, A. Sell, and A. Leitenstorfer, Opt. Lett. 35, 3282 (2010).

${ }^{20}$ A. M. Weiner, D. E. Leaird, J. S. Patel, and J. R. Wullert, Opt. Lett. 15, 326 (1990).

${ }^{21}$ E. Zeek, K. Maginnis, S. Backus, U. Russek, M. Murnane, G. Mourou, H. Kapteyn, and G. Vdovin, Opt. Lett. 24, 493 (1999).

${ }^{22}$ M. A. Dugan, J. X. Tull, and W. S. Warren, J. Opt. Soc. Am. B 14, 2348 (1997).

${ }^{23}$ D. A. Flickinger, R. N. Coffee, G. N. Gibson, and T. C. Weinacht, Appl. Opt. 45, 6187 (2006). 